\title{
Chattering-Free Time Scale Separation Sliding Mode Control Design with Application to Power System Chaos Suppression
}

\author{
Junkang Ni, Ling Liu, Chongxin Liu, and Xiaoyu Hu \\ State Key Laboratory of Electrical Insulation and Power Equipment, School of Electrical Engineering, \\ Xian Jiaotong University, Xian 710049, China \\ Correspondence should be addressed to Junkang Ni; max12391@126.com
}

Received 23 December 2015; Revised 28 January 2016; Accepted 3 February 2016

Academic Editor: Yan-Wu Wang

Copyright (C) 2016 Junkang Ni et al. This is an open access article distributed under the Creative Commons Attribution License, which permits unrestricted use, distribution, and reproduction in any medium, provided the original work is properly cited.

This paper presents a novel chattering-free sliding mode control method for a class of disturbed nonlinear systems, which achieves fast and exact disturbance estimation, eliminates chattering, and recovers the performance of nominal system and nominal control input. The proposed approach combines time scale separation design and sliding mode control. Different from the existing disturbance estimation based sliding mode control methods, the proposed scheme achieves fast and exact disturbance estimation through time scale separation and eliminates discontinuous switching term, thereby achieving good chattering alleviation effect and providing good transient response. The proposed control method is applied to suppress chaos in power system and simulation results confirm the effectiveness and robustness of proposed control scheme and highlight the advantages of the proposed control scheme over the existing disturbance estimation based sliding mode control methods in terms of chattering alleviation effect and transient response.

\section{Introduction}

The power system is a complex nonlinear dynamical system and when the power system operates near its stability boundary, chaotic oscillation may occur. The soaring growth of power consumption pushes the power system to operate near its stability boundary. In heavily loaded power system, parameter variations [1], time delay [2], and external disturbances [3-5] can induce chaos. Chaotic oscillation is an undesirable phenomenon for power system. It will result in voltage collapse, rotor angle instability, frequency oscillation, and even catastrophic blackout [6-8]. Therefore, it is urgently needed to find an effective way for chaos suppression in power system.

Rapid development of modern control theory provides many advanced methods for chaos control, such as feedback control $[9,10]$, passive control [11], sliding mode control $[12,13]$, finite time control [14], adaptive control [15], fuzzy control [16-18], and neural network control $[19,20]$. Some of them have been applied to suppress chaos in power system. Among these control methods, sliding mode control is particularly appealing due to its simple implementation, good transient performance, and robustness against parameter uncertainties and external disturbances. Due to these attractive features, sliding mode control has been applied to soft landing control [21], trajectory tracking [22], motor speed control [23], power converter control [24], power system control [25], and so forth. However, chattering problem caused by parasitic dynamics and nonideal switching obstructs the development and application of sliding mode control. Chattering reduces convergence accuracy, increases power losses, excites high frequency dynamics, and even causes damage to controller and system.

Many methods have been proposed to eliminate chattering. The most frequently used method is to replace discontinuous switching control with continuous saturation control input, which is called boundary layer approach. Examples of works that employed boundary layer method to attenuate chattering include those of Burton and Zinober [26], Chen et al. [27], Wu et al. [28], and Qiao et al. [29]. However, this method ensures convergence to boundary layer rather than sliding surface, which may result in the existence of steady state error. Moreover, it is difficult to select an appropriate 
controller parameter when the disturbance is unknown. In fact, the disturbance in many practical systems, for example, power system load disturbance, is difficult to obtain due to the complexity and the unpredictability of the disturbance. For unknown disturbance, a conservative large switching gain should be selected to counteract its influence and the width of boundary layer should increase to reduce chattering caused by large switching gain. However, thicker boundary layer results in larger convergence error. Therefore, a compromise between chattering, robustness, and control accuracy should be made when selecting the width of boundary layer and switching gain.

Disturbance estimation based sliding mode control can overcome the drawback of boundary layer method. Generally speaking, the existing disturbance estimation methods can be divided into two categories. The first category employs adaptive law to estimate the upper bound of disturbance. In [30], a chattering-free adaptive sliding mode was designed for synchronization of two uncertain chaotic systems. Lin and Chiu [31] presented a simple adaptive sliding mode algorithm to control the position of disturbed PM synchronous servo motor drive. An adaptive PID sliding mode control was considered for a class of nonlinear systems in [32]. The principle of adaptive sliding mode control is adjusting the switching gain according to the distance from switching surface. However, due to the existence of sensor noise and finite frequency switching action in practical system, the system state cannot strictly stay on the sliding surface, which results in unbounded increment of switching gain. Unbounded growth of switching gain will result in serious chattering [33]. This problem is called parameter drift problem [34]. The other problem in adaptive sliding mode control is referred to as overadaptation problem, which is caused by overestimation of actual disturbance [35].

The second category is referred to as disturbance observer based sliding mode control. Zhang et al. [36] designed an adaptive sliding mode disturbance observer to alleviate chattering. Yang et al. [37] utilized a nonsingular terminal sliding mode control based on finite-time disturbance observer to attenuate uncertainties and reduce chattering. Using nonlinear disturbance observer, M. Chen and W.-H. Chen [38] developed a sliding mode control to avoid chattering and stabilize a class of nonlinear systems. In [39], an improved fuzzy disturbance observer based predictive sliding mode control was presented to enhance composite disturbance rejection performance and eliminate chattering. However, observer-based sliding mode control requires the transient response of the observer should be significantly shorter than system's transient response. Otherwise, robustness problem and chattering problem may arise due to overestimation or underestimation of disturbance during long observation phase. One method to achieve fast disturbance estimation is to adopt high gain observer. However, measurement noise and unmodeled dynamics are amplified by high gain, which makes it impossible to be implemented in practical system $[40,41]$. Therefore, it is necessary to find another fast disturbance estimation method to eliminate chattering in sliding mode.
Time scale separation design proposed by Chakrabortty and Arcak [42] can shorten the disturbance estimation time and achieve performance recovery of nominal system. The philosophy of time scale separation design is to use one filter to estimate unknown disturbance over a fast time scale and the other filter to drive the control input to the nominal one over an intermediate time scale. Afterwards, the control input will recover the nominal system performance over a slow time scale.

Motivated by aforementioned discussion, this paper proposes time scale separation sliding mode control to avoid chattering in sliding mode control and achieve nominal system performance recovery. Instead of employing high gain disturbance observer, the proposed control strategy achieves fast and exact disturbance estimation through time scale separation. The main idea of the proposed control scheme is as follows. First, a sliding mode control is designed for nominal system. Subsequently, three-time-scale separation is employed to achieve fast and exact disturbance estimation over fast time scale, nominal control input recovery over intermediate time scale, and nominal system performance recovery over slow time scale. Finally, the discontinuous switching control is replaced by the estimated disturbance. The proposed control scheme is applied to suppress chaos in power system, recover the nominal system performance, and eliminate the influence of load disturbance. In comparison with existing results of disturbance estimation based sliding mode control, the main features of proposed control scheme can be summarized as follows:

(i) There is no switching term in the proposed control scheme, and as a result, chattering can be eliminated completely.

(ii) The exact disturbance estimation can be achieved within a much shorter time (usually in a millisecond), which avoids the robustness and chattering problem in estimation process.

(iii) The performance of nominal control input and nominal system can be recovered.

The rest of this paper is organized as follows. The problems in existing disturbance estimation based sliding mode control are presented in Section 2. Section 3 presents time scale separation sliding mode control design. In Section 4, the proposed control method is applied to suppress chaos in power system and numerical simulation is provided to show the effectiveness, the robustness, and the superiority of proposed control scheme. Finally, the conclusion is drawn in Section 5.

\section{Problem Formulation and Motivation}

Consider a class of nonlinear systems:

$$
\begin{aligned}
& \dot{x}=f(x)+g(x)\left(u_{1}+d\right), \\
& y=h(x),
\end{aligned}
$$

where $x \in R^{n}$ is state variables, $u_{1}$ is the control input, $f(x)$ and $g(x)$ are known smooth functions of $x, d$ denotes 
matched disturbance, and $y$ is the output function of the system satisfying the following condition:

$$
\alpha(x)=L_{g} h(x)=\frac{\partial h}{\partial x} g(x) \neq 0 .
$$

Then, time derivative of system output $y$ can be expressed as

$$
\dot{y}=L_{f} h(x)+\alpha(x)\left(u_{1}+d\right) .
$$

Assumption 1. It is assumed that the disturbance $d$ is unknown, slowly time-varying, and bounded. That is,

$$
\begin{aligned}
\|d\| & \leq D, \\
\dot{d} & =0,
\end{aligned}
$$

where $D$ is an unknown positive constant.

Remark 2. For many systems, the disturbance is slowly varying. For example, in power system normal operation, the variation of active and reactive load can be considered as slowly varying disturbance.

The control problem can be formulated as designing control input for system (1) such that system output $y$ can be stabilized to its desired output value $y_{d}$ asymptotically.

Since the bound of disturbance is unknown, switching gain needs to be selected sufficiently large to ensure reaching condition. However, high switching control gain will result in serious chattering. Therefore, disturbance estimation is needed to determine the switching gain so that reaching condition is satisfied and chattering can be minimized. In general, the existing results of disturbance estimation based sliding mode control can be divided into two categories, that is, adaptive sliding mode control and disturbance observer based sliding mode control. In this paper, the adaptive sliding mode proposed in [43] and nonlinear disturbance observer based sliding mode control presented in [38] are taken as representatives to demonstrate the problems in existing disturbance estimation based sliding mode control.

2.1. Adaptive Sliding Mode Control. An effective method to estimate disturbance and alleviate chattering would be adaptive sliding mode control. The sliding surface of adaptive sliding mode control can be constructed as

$$
s=y-y_{d},
$$

where $y_{d}$ is desired constant output; that is, $\dot{y}_{d}=0$.

The system state will reach the sliding surface asymptotically if the control input of adaptive sliding mode control is

$$
u_{1}=\frac{1}{\alpha(x)}\left(-k s-L_{f} h(x)\right)-\widehat{d} \operatorname{sign}(s \cdot \alpha(x)),
$$

where $\widehat{d}$ is the adaptive estimation for the upper bound of disturbance $d$.

According to [43], the adaptation law can be selected as

$$
\begin{aligned}
& \dot{\hat{d}}=-\mu \widehat{d}+|s||\alpha(x)|, \\
& \dot{\mu}=-\beta \mu .
\end{aligned}
$$

The stability proof is very simple and straightforward and it is omitted here. Interested reader can refer to [43] for detailed proof process.

Remark 3. In (7), a negative feedback term $-\mu \widehat{d}$ is introduced to guarantee the boundedness of estimated upper bound of disturbance, thereby reducing chattering. However, overadaptation problem [35] and discontinuous switching term still exist in adaptive sliding mode control design. Therefore, this adaptive sliding mode control scheme cannot eliminate chattering.

2.2. Nonlinear Disturbance Observer Based Sliding Mode Control. Another alternative approach to avoid chattering and attenuate disturbance is to observe disturbance via nonlinear disturbance observer. The nonlinear disturbance observer can be constructed as

$$
\begin{aligned}
& \widehat{d}=z+p(x), \\
& \dot{z}=-l(x) g(x) z-l(x)\left(f(x)+g(x)\left(u_{1}+p(x)\right)\right),
\end{aligned}
$$

where $l(x)$ is nonlinear disturbance observer gain and satisfies $l(x)=\partial p(x) / \partial x$ and $l(x) g(x)>0, \widehat{d}$ is observed disturbance.

The error between actual disturbance and observed disturbance can be described as

$$
\widetilde{d}=d-\widehat{d} .
$$

Utilizing Assumption 1 and disturbance observer equation (9), the differential equation of the error $\widetilde{d}$ can be obtained as

$$
\dot{\tilde{d}}=-l(x) g(x) \tilde{d} .
$$

It can be derived from (11) that the disturbance observer estimation error will exponentially converge to zero as long as $l(x) g(x)>0$ holds.

Employing the same sliding surface as adaptive sliding mode control and the control law can be designed as

$$
u_{1}=\frac{1}{\alpha(x)}\left(-k s-L_{f} h(x)\right)-\widehat{d}-\frac{s \alpha(x)}{4 l(x) g(x)} .
$$

The system state will reach the sliding surface asymptotically. The stability proof is very simple and straightforward and it is omitted here. Interested reader can refer to [38] for detailed proof process.

Remark 4. For most observers, a certain amount of time is required to observe the disturbance. During observation phase, the disturbance value cannot be obtained exactly and the estimated disturbance may be so large that it will result in serious chattering or so small that the robustness may be lost. One approach to achieve fast disturbance estimation is to use high gain observer. However, sensitivity to measurement noise and unmodeled dynamics renders it impossible to be applied in practical systems $[40,41]$. Therefore, it is necessary to find another method to achieve exact disturbance estimation within a short time to achieve better transient response. 
This motivates us to design a novel disturbance estimation based sliding mode control to achieve fast and exact disturbance estimation so as to eliminate chattering and improve transient response.

\section{Time Scale Separation Sliding Mode Control Design}

In this section, a time scale separation sliding mode controller is designed to achieve fast and exact disturbance estimation and eliminate chattering in sliding mode for perturbed system (1).

The nominal system of perturbed system (1) can be expressed as

$$
\begin{aligned}
& \dot{x}=f(x)+g(x) u, \\
& y=h(x),
\end{aligned}
$$

where $u$ is control input for nominal system.

Constructing the same sliding surface as (5) and the control law can be designed as

$$
u=\frac{1}{\alpha(x)}\left(-k s-L_{f} h(x)\right),
$$

where $k$ is a positive constant.

Theorem 5. For nominal system (13), if the control input is designed as (14), the output of system (13) can converge to its desired output value $y_{d}$ asymptotically.

Proof. Consider the following Lyapunov candidate:

$$
V=\frac{1}{2} s^{2}
$$

Time derivative of Lyapunov function $V$ yields

$$
\dot{V}=s \dot{s}=-k s^{2} \leq 0
$$

Therefore, the control input (14) can achieve system output asymptotically stabilization to its desired output value.

Remark 6. This design method utilizes continuous term $-k s$ to replace signum function, thereby eliminating chattering. In comparison with boundary layer method, related studies have shown that this design has smaller steady-state error, shorter hitting time, and stronger robustness [44] and solves the large gain issue in the initial stage [45].

Now, let us consider the perturbed system (1). A firstorder filter is constructed to estimate the system uncertainty:

$$
\dot{\hat{y}}=L_{f} h(x)-\frac{\hat{y}-y}{\mu}, \quad y(0)=\hat{y}(0) .
$$

Define a new variable $l=(\widehat{y}-y) / \mu$, and subtracting (3) from (17) yields

$$
\mu \dot{l}=-l-\alpha(x)\left(u_{2}+d\right), \quad l(0)=0,
$$

where $\mu$ is a small positive parameter and $u_{2}$ is redesigned control input for perturbed system (1).

According to singular perturbation theory [46], since $\mu$ is small, the convergence speed of $l$ is faster than state variables of system (1). After $l$ converges to its quasisteady value, the estimate for uncertainty can be expressed as

$$
d=-\alpha(x)^{-1} l-u_{2}
$$

Using this estimate, we construct the other filter:

$$
\eta \dot{w}=\alpha(x)^{-1} l+u
$$

where $\eta$ is the other small parameter and $u$ is control input for nominal system. The control input for uncertain system (1) can be designed as

$$
u_{2}=u+w
$$

Remark 7. In order to recover the nominal system performance, the following control law can be designed for perturbed system (1):

$$
u_{2}^{\prime}=u-d
$$

Since the filter (20) uses the estimate generated by filter (18), the convergence speed of $l$ must be faster than that of $w$. Therefore, filter parameters $\mu$ and $\eta$ must satisfy $\mu \ll \eta$.

Taking the correlation of two time scales into consideration, we assign

$$
\begin{aligned}
& \mu=\varepsilon_{1} \varepsilon_{2}, \\
& \eta=\varepsilon_{1},
\end{aligned}
$$

where $\varepsilon_{1}$ and $\varepsilon_{2}$ are independent small parameters.

According to design procedure described above, the block diagram of the proposed control scheme is illustrated in Figure 1.

Next, we will show this design can recover the performance of nominal system and the control input.

Theorem 8. For any compact sets $\Omega_{x}, \Omega_{w}, \Omega_{s}$, and $\Omega_{d}$ of initial conditions $x(0), w(0), s(0)$, and $d(0)$, there exists a positive constant $\varepsilon_{2}^{*}$ such that for all $k>0, \varepsilon_{1}>0$, and $0<\varepsilon_{2}<\varepsilon_{2}^{*}$ and for all $x(0) \in \Omega_{x}, w(0) \in \Omega_{w}, s(0) \in \Omega_{s}$, and $d(0) \in \Omega_{d}$, the control input (21) can achieve nominal system performance and nominal control input recovery and guarantee that the system output $y$ converges to its desired output value $y_{d}$ asymptotically.

Proof. Define new variables $\widetilde{w}$ and $\widetilde{l}$ to represent the error between the designed control input (21) and nominal control input (22) and the error between estimated system disturbance and actual system disturbance, respectively. The expression for new variables can be given as

$$
\begin{aligned}
\widetilde{w} & =u_{2}-u_{2}^{\prime}=w+u-(u-d)=w+d, \\
\widetilde{l} & =-l-\alpha(x) u_{2}-\alpha(x) d \\
& =-l-\alpha(x) u-\alpha(x)(w+d) \\
& =-l-\alpha(x) u-\alpha(x) \widetilde{w} .
\end{aligned}
$$




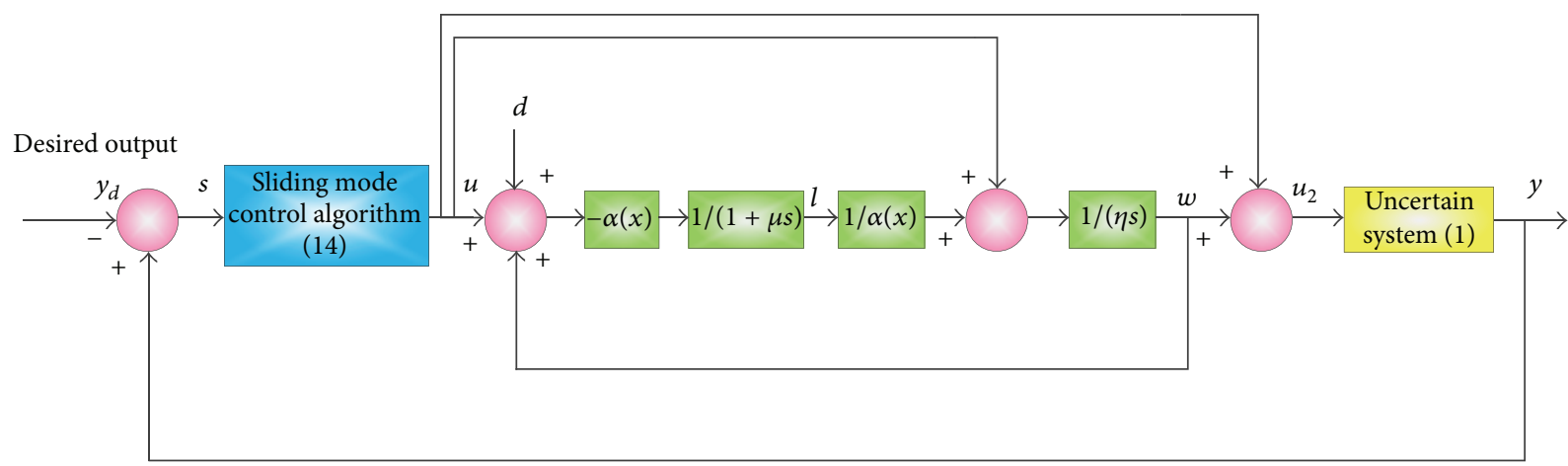

FIGURE 1: Block diagram of time scale separation sliding mode control for uncertain system (1).

Noting that the disturbance $d$ satisfies Assumption 1, we get

$$
\begin{aligned}
& \dot{\tilde{w}}=\dot{w}, \\
& \dot{\tilde{l}}=-\dot{l}-\frac{\mathrm{d}(\alpha(x) u)}{\mathrm{d} t}-\frac{\mathrm{d}(\alpha(x) \widetilde{w})}{\mathrm{d} t} .
\end{aligned}
$$

The Lyapunov function candidate can be defined as

$$
W=\frac{1}{2} s^{2}+\frac{1}{2} \widetilde{l}^{2}+\frac{1}{2} \widetilde{w}^{2} .
$$

Considering (16), (18), (20), and (23)-(27), we have

$$
\begin{aligned}
\dot{W}= & s \dot{s}+\widetilde{l} \dot{\vec{l}}+\widetilde{w} \dot{\tilde{w}} \\
= & -k\|s\|^{2}-\frac{1}{\varepsilon_{1} \varepsilon_{2}}\|\tilde{l}\|^{2}-\widetilde{l} \frac{\mathrm{d}(\alpha(x) u)}{\mathrm{d} t}-\widetilde{l} \frac{\mathrm{d}(\alpha(x) \widetilde{w})}{\mathrm{d} t} \\
& +\frac{1}{\varepsilon_{1}}(w+d)\left(\alpha(x)^{-1} l+u\right) .
\end{aligned}
$$

Since $l(0)=0$, we have

$$
\begin{aligned}
\tilde{l}(0)= & -l(0)-\left(-k s(0)-L_{f} h(x(0))\right) \\
& -\alpha(x(0))(w(0)+d(0))
\end{aligned}
$$

which means that for any compact sets $\Omega_{x}, \Omega_{w}, \Omega_{s}$, and $\Omega_{d}$, one may find a corresponding set $\Omega_{\tilde{l}}$ of initial condition $\tilde{l}(0)$ and a set $\Omega_{c}$ of $W$, such that $\Omega_{x} \times \Omega_{w} \times \Omega_{s} \times \Omega_{d} \times$ $\Omega_{\tilde{I}} \subseteq \Omega_{c}$. Since $\alpha(x)$ and $u$ are at least $C^{1}$ function, there exist positive numbers $L_{1}-L_{4}$ such that, on the set $\Omega_{c}$, the following inequalities hold:

$$
\begin{aligned}
\|\alpha(x)\| & \leq L_{1}, \\
\left\|\frac{\mathrm{d}(\alpha(x) u)}{\mathrm{d} s}\right\| & \leq L_{2}, \\
\left\|\frac{\mathrm{d}(\alpha(x))}{\mathrm{d} t}\right\| & \leq L_{3}, \\
\left\|\alpha(x)^{-1}\right\| & \leq L_{4} .
\end{aligned}
$$

Using (31)-(34), the following inequalities can be obtained:

$$
\begin{aligned}
& \left\|\frac{\mathrm{d}(\alpha(x) \widetilde{w})}{\mathrm{d} t}\right\| \leq L_{5}\|\widetilde{w}\|+L_{6}\|\tilde{\imath}\|, \\
& \left\|\frac{\mathrm{d}(\alpha(x) u)}{\mathrm{d} t}\right\| \leq L_{7}\|s\|+L_{8}\|\widetilde{w}\| .
\end{aligned}
$$

Detailed derivation for (35) and (36) can be found in the appendix.

Substituting (35) and (36) into (29) and utilizing Young's inequality and (34), one has

$\dot{W}$

$$
\begin{aligned}
& \leq-k\|s\|^{2}-\frac{1}{\varepsilon_{1} \varepsilon_{2}}\|\bar{l}\|^{2}+\|\tilde{l}\|\left\|\frac{\mathrm{d}(\alpha(x) u)}{\mathrm{d} t}\right\| \\
& +\|\bar{l}\|\left\|\frac{\mathrm{d}(\alpha(x) \widetilde{w})}{\mathrm{d} t}\right\| \\
& +\frac{1}{\varepsilon_{1}}(w+d)\left(\alpha(x)^{-1} l+u+w+d-w-d\right) \\
& \leq-k\|s\|^{2}-\frac{1}{\varepsilon_{1} \varepsilon_{2}}\|\tilde{l}\|^{2}+L_{7}\|s\|\|\bar{l}\|+L_{8}\|\widetilde{w}\|\|\bar{l}\| \\
& +L_{5}\|\widetilde{w}\|\|\widetilde{l}\|+L_{6}\|\widetilde{l}\|^{2} \\
& +\frac{1}{\varepsilon_{1}}(w+d)\left(\alpha(x)^{-1} l+u_{2}+d\right)-\frac{1}{\varepsilon_{1}}(w+d)^{2} \\
& \leq-k\|s\|^{2}-\frac{1}{\varepsilon_{1} \varepsilon_{2}}\|\bar{l}\|^{2}+\frac{L_{7} \lambda_{1}}{2}\|s\|^{2}+\frac{L_{7}}{2 \lambda_{1}}\|\bar{l}\|^{2} \\
& +\frac{L_{8} \lambda_{2}}{2}\|\widetilde{w}\|^{2}+\frac{L_{8}}{2 \lambda_{2}}\|\widetilde{l}\|^{2}+\frac{L_{5} \lambda_{3}}{2}\|\widetilde{w}\|^{2}+\frac{L_{5}}{2 \lambda_{3}}\|\vec{l}\|^{2} \\
& +L_{6}\|\bar{l}\|^{2}+\frac{\lambda_{4}}{2 \varepsilon_{1}}(w+d)^{2} \\
& +\frac{1}{2 \varepsilon_{1} \lambda_{4}}\left(\alpha(x)^{-1} l+u_{2}+d\right)^{2}-\frac{1}{\varepsilon_{1}}(w+d)^{2} \\
& \leq-k\|s\|^{2}-\frac{1}{\varepsilon_{1} \varepsilon_{2}}\|\bar{l}\|^{2}+\frac{L_{7} \lambda_{1}}{2}\|s\|^{2}+\frac{L_{7}}{2 \lambda_{1}}\|\tilde{l}\|^{2} \\
& +\frac{L_{8} \lambda_{2}}{2}\|\widetilde{w}\|^{2}+\frac{L_{8}}{2 \lambda_{2}}\|\widetilde{l}\|^{2}+\frac{L_{5} \lambda_{3}}{2}\|\widetilde{w}\|^{2}+\frac{L_{5}}{2 \lambda_{3}}\|\vec{l}\|^{2}
\end{aligned}
$$




$$
\begin{aligned}
& +L_{6}\|\tilde{l}\|^{2}+\frac{\lambda_{4}}{2 \varepsilon_{1}}\|\widetilde{w}\|^{2}+\frac{L_{4}^{2}}{2 \varepsilon_{1} \lambda_{4}}\|\tilde{l}\|^{2}-\frac{1}{\varepsilon_{1}}\|\widetilde{w}\|^{2} \\
= & -\left(k-\frac{L_{7} \lambda_{1}}{2}\right)\|s\|^{2} \\
& -\left(\frac{1}{\varepsilon_{1} \varepsilon_{2}}-\frac{L_{7}}{2 \lambda_{1}}-\frac{L_{8}}{2 \lambda_{2}}-\frac{L_{5}}{2 \lambda_{3}}-L_{6}-\frac{L_{4}^{2}}{2 \varepsilon_{1} \lambda_{4}}\right)\|\tilde{l}\|^{2} \\
& -\left(\frac{1}{\varepsilon_{1}}-\frac{L_{8} \lambda_{2}}{2}-\frac{L_{5} \lambda_{3}}{2}-\frac{\lambda_{4}}{2 \varepsilon_{1}}\right)\|\widetilde{w}\|^{2},
\end{aligned}
$$

where $\lambda_{1}, \lambda_{2}, \lambda_{3}$, and $\lambda_{4}$ are arbitrary positive numbers. Without loss of generality, we select

$$
\begin{aligned}
& \lambda_{1}=\frac{k}{L_{7}}, \\
& \lambda_{2}=\frac{1}{3 L_{8} \varepsilon_{1}}, \\
& \lambda_{3}=\frac{1}{3 L_{5} \varepsilon_{1}}, \\
& \lambda_{4}=\frac{1}{3} .
\end{aligned}
$$

Define

$$
\begin{aligned}
& \varepsilon_{2}^{*} \\
& =\frac{1}{\varepsilon_{1}\left(L_{7}^{2} / 2 k+3 L_{8}^{2} \varepsilon_{1} / 2+3 L_{5}^{2} \varepsilon_{1} / 2+L_{6}+3 L_{4}^{2} / 2 \varepsilon_{1}\right)} .
\end{aligned}
$$

Substitute (38) into (37) and we have

$$
\begin{aligned}
\dot{W} & \leq-\frac{k}{2}\|s\|^{2} \\
& -\left(\frac{1}{\varepsilon_{1} \varepsilon_{2}}-\left(\frac{L_{7}^{2}}{2 k}+\frac{3 L_{8}^{2} \varepsilon_{1}}{2}+\frac{3 L_{5}^{2} \varepsilon_{1}}{2}+L_{6}+\frac{3 L_{4}^{2}}{2 \varepsilon_{1}}\right)\right) \\
& \cdot\|\tilde{l}\|^{2}-\frac{1}{2 \varepsilon_{1}}\|\widetilde{w}\|^{2} .
\end{aligned}
$$

It can be concluded that if the parameters can be selected to satisfy $k>0, \varepsilon_{1}>0$, and $0<\varepsilon_{2}<\varepsilon_{2}^{*}$, the time derivative of Lyapunov function $W$ is negative definite. Therefore, the nominal system performance and nominal control input can be recovered and the system output $y$ can converge to its desired output value $y_{d}$ asymptotically.

Remark 9. Here, we use time scale separation design method to estimate the unknown disturbance and replace discontinuous switching control with continuous one. The whole design process requires no discontinuous control term, so chattering phenomenon can be avoided.

\section{Application to Suppress Chaos in Power System}

The power system model studied in this paper is shown in Figure 2, which was introduced in [47] as a benchmark model



FIgURE 2: Studied power system model.

for voltage stability study. The model is constituted by two generator buses and one load bus. One generator bus is slack bus and the other generator can be modeled by classical swing equation. The load bus consists of constant PQ load in parallel with induction motor whose model is taken from Walve [48]. The dynamics of the power system model are governed by the following four differential equations [47]:

$$
\begin{aligned}
& \dot{\delta_{m}}=\omega \\
& M \dot{\omega}=-d_{m} \omega+P_{m}+E_{m} Y_{m} V \sin \left(\delta-\delta_{m}-\theta_{m}\right) \\
& \quad+E_{m}^{2} Y_{m} \sin \theta_{m} \\
& K_{q w} \dot{\delta}=-K_{q v 2} V^{2}-K_{q v} V+E_{0}^{\prime} Y_{0}^{\prime} V \cos \left(\delta+\theta_{0}^{\prime}\right) \\
& \quad+E_{m} Y_{m} V \cos \left(\delta-\delta_{m}+\theta_{m}\right)-\left(Y_{0}^{\prime} \cos \theta_{0}^{\prime}\right. \\
& \left.\quad+Y_{m} \cos \theta_{m}\right) V^{2}-Q_{0}-Q_{1} \\
& T K_{q w} K_{p v} \dot{V}=K_{p w} K_{q v 2} V^{2}+\left(K_{p w} K_{q v}-K_{q w} K_{p v}\right) V \\
& \quad+K_{q w}\left(-E_{0}^{\prime} Y_{0}^{\prime} V \sin \left(\delta+\theta_{0}^{\prime}\right)\right. \\
& \quad-E_{m} Y_{m} V \sin \left(\delta-\delta_{m}+\theta_{m}\right) \\
& \left.\quad+\left(Y_{0}^{\prime} \sin \theta_{0}^{\prime}+Y_{m} \sin \theta_{m}\right) V^{2}-P_{0}-P_{1}\right) \\
& \quad-K_{p w}\left(E_{0}^{\prime} Y_{0}^{\prime} V \cos \left(\delta+\theta_{0}^{\prime}\right)\right. \\
& +E_{m} Y_{m} V \cos \left(\delta-\delta_{m}+\theta_{m}\right) \\
& \left.\quad-\left(Y_{0}^{\prime} \cos \theta_{0}^{\prime}+Y_{m} \cos \theta_{m}\right) V^{2}-Q_{0}-Q_{1}\right)
\end{aligned}
$$

where $\delta_{m}$ and $\omega$ denote generator angle and frequency deviation, respectively; $M$ is generator inertia; $d_{m}$ refers to damping coefficient and $P_{m}$ is mechanical power; $Y_{m}$ and $\theta_{m}$ are admittance and impedance angle of transmission line; $E_{m}$ stands for the magnitude of generator voltage; $\delta$ and $V$ are phase angle and magnitude of load voltage; $P_{1}$ and $Q_{1}$ are real and reactive power demand of constant PQ load; 

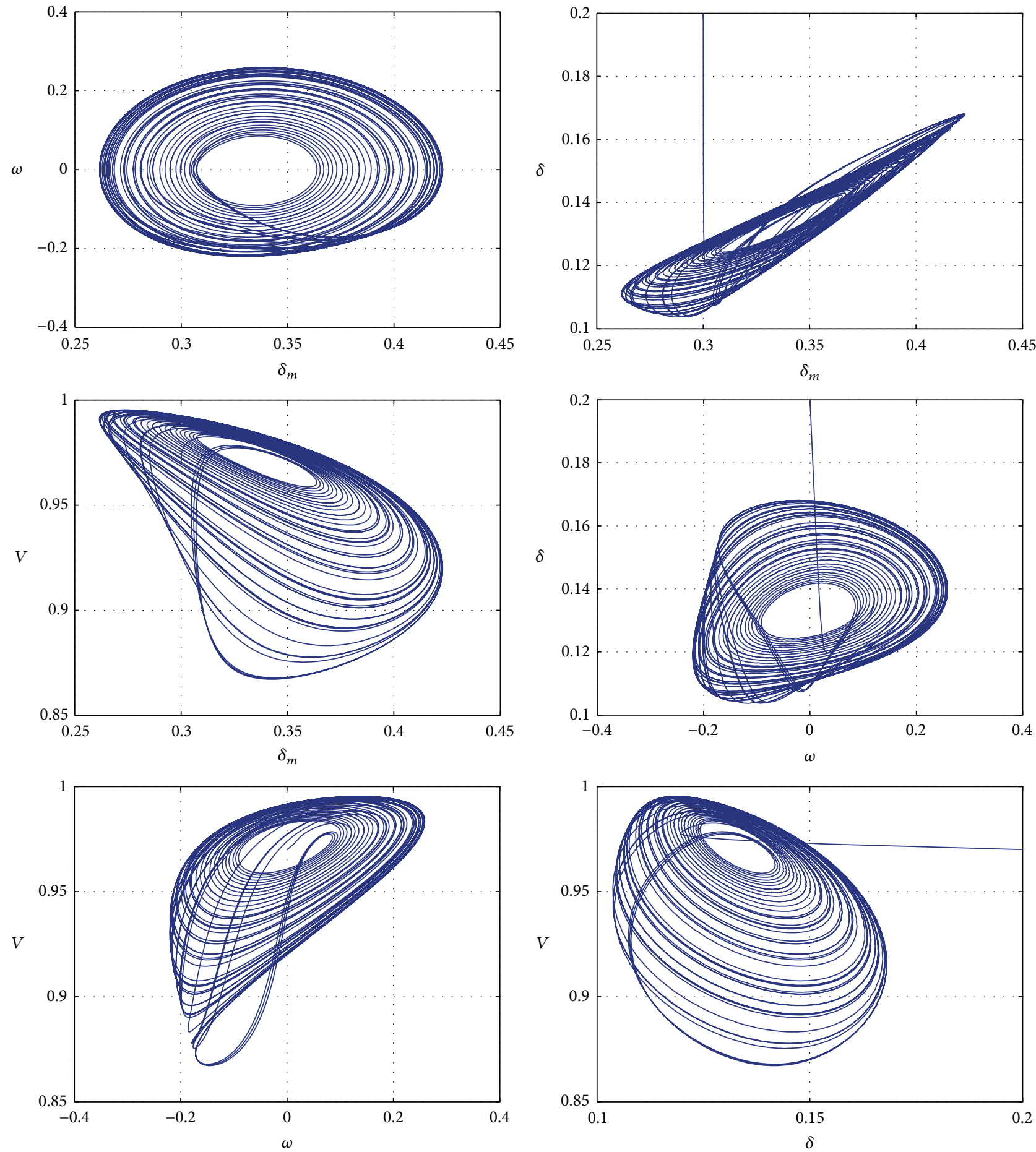

FIgURE 3: Phase portraits of power system.

and $P_{0}$ and $Q_{0}$ are the constant real and reactive power for the induction motor. $K_{p w}, K_{p v}, K_{q w}, K_{q v}$, and $K_{q v 2}$ are constants associated with the induction motor; $E_{0}^{\prime}, Y_{0}^{\prime}$, and $\theta_{0}^{\prime}$ are Thevenin equivalent circuit values with respect to $E_{0}$, $Y_{0}$, and $\theta_{0}$, which can be described by the following three equations: 



FIGURE 4: Time domain waveform of power system.

$$
\begin{aligned}
& E_{0}^{\prime}=\frac{E_{0}}{\sqrt{1+C^{2} Y_{0}^{-2}-2 C Y_{0}^{-1} \cos \theta_{0}}}, \\
& Y_{0}^{\prime}=Y_{0} \sqrt{1+C^{2} Y_{0}^{-2}-2 C Y_{0}^{-1} \cos \theta_{0}}, \\
& \theta_{0}^{\prime}=\theta_{0}+\tan ^{-1}\left(\frac{C Y_{0}^{-1} \sin \theta_{0}}{1-C Y_{0}^{-1} \cos \theta_{0}}\right),
\end{aligned}
$$

where $E_{0}$ is voltage magnitude of an infinite system; $Y_{0}$ and $\theta_{0}$ are termed as admittance and impedance angle of transmission line.

In our simulation, parameter values for network and generator are $E_{m}=1, Y_{m}=5, P_{m}=1, \theta_{m}=-5.0, E_{0}^{\prime}=2.5$, $Y_{0}^{\prime}=8, \theta_{0}^{\prime}=-12, P_{m}=1, d_{m}=0.05$, and $M=0.3$. Load parameter values are adopted as $K_{p w}=0.4, K_{q v 2}=2.1$, $K_{q w}=-0.03, K_{q v}=-2.8, K_{p v}=0.3, T=8.5, P_{0}=0.6$, $Q_{0}=1.3$, and $P_{1}=0$. All values are in per unit except for angles, which are in degrees.

Parameter $Q_{1}$ plays an important role in the dynamics of chaos and voltage collapse. When $Q_{1}$ varies to 11.377, chaotic attractor emerges and the phase portraits of chaotic attractor are shown in Figure 3. As can be seen from Figure 3, chaotic behavior of the power system is presented completely. Figure 4 displays time domain waveform of chaotic power system. It can be observed from Figure 4 that the time responses of state variables show so aperiodic and irregular oscillatory behaviors that it is impossible to predict their trajectories after a long period of time. Wolf algorithm [49] is adopted to calculate the Lyapunov exponents and the computation result at $Q_{1}=11.377$ is

$$
\begin{aligned}
& \left(\lambda_{1}, \lambda_{2}, \lambda_{3}, \lambda_{4}\right) \\
& \quad=(0.3088,-0.0073,-4.1459,-66.1876) .
\end{aligned}
$$

There is one positive Lyapunov exponent in this system. All the aforementioned analysis proves the existence of chaos in the power system.

If no control action is activated, chaotic attractor may be broken and it will result in voltage collapse. Voltage collapse is one of the main causes for catastrophic blackout. Therefore, it is vitally important to study control method for chaos suppression in power system to avoid voltage collapse.

It is reported that chaotic oscillation can be suppressed by reactive power control [50-52]. In power system, reactive compensator devices, such as static var compensator (SVC) and static synchronous compensator (STATCOM), can provide flexible reactive power control. In this paper, reactive power controller is employed to suppress chaos in power system and the controlled power system model can be expressed as

$$
\begin{aligned}
& \dot{\delta_{m}}=\omega \\
& M \dot{\omega}=-d_{m} \omega+P_{m}+E_{m} Y_{m} V \sin \left(\delta-\delta_{m}-\theta_{m}\right) \\
& \quad+E_{m}^{2} Y_{m} \sin \theta_{m}, \\
& K_{q w} \dot{\delta}=-K_{q v 2} V^{2}-K_{q v} V+E_{0}^{\prime} Y_{0}^{\prime} V \cos \left(\delta+\theta_{0}^{\prime}\right) \\
& \quad+E_{m} Y_{m} V \cos \left(\delta-\delta_{m}+\theta_{m}\right)-\left(Y_{0}^{\prime} \cos \theta_{0}^{\prime}\right. \\
& \left.\quad+Y_{m} \cos \theta_{m}\right) V^{2}-Q_{0}-Q_{1}-u-\Delta Q \\
& T K_{q w} K_{p v} \dot{V}=K_{p w} K_{q v 2} V^{2}+\left(K_{p w} K_{q v}-K_{q w} K_{p v}\right) V \\
& \quad+K_{q w}\left(-E_{0}^{\prime} Y_{0}^{\prime} V \sin \left(\delta+\theta_{0}^{\prime}\right)\right.
\end{aligned}
$$




$$
\begin{aligned}
& -E_{m} Y_{m} V \sin \left(\delta-\delta_{m}+\theta_{m}\right) \\
& \left.+\left(Y_{0}^{\prime} \sin \theta_{0}^{\prime}+Y_{m} \sin \theta_{m}\right) V^{2}-P_{0}-P_{1}\right) \\
& -K_{p w}\left(E_{0}^{\prime} Y_{0}^{\prime} V \cos \left(\delta+\theta_{0}^{\prime}\right)\right. \\
& +E_{m} Y_{m} V \cos \left(\delta-\delta_{m}+\theta_{m}\right) \\
& -\left(Y_{0}^{\prime} \cos \theta_{0}^{\prime}+Y_{m} \cos \theta_{m}\right) V^{2}-Q_{0}-Q_{1}-u \\
& -\Delta Q) .
\end{aligned}
$$

Comparing the mathematical model of controlled power system (44) with the form presented in (1), we have

$$
\begin{aligned}
& x=\left[\delta_{m}, \omega, \delta, V\right], \\
& f(x)=\left[f_{1}, f_{2}, f_{3}, f_{4}\right]^{T}, \\
& g(x)=\left[\begin{array}{llll}
0 & 0 & -\frac{1}{K_{q w}} & \frac{K_{p w}}{T K_{q w} K_{p v}}
\end{array}\right]^{T}, \\
& y=V, \\
& d=\Delta Q,
\end{aligned}
$$

where

$$
\begin{aligned}
f_{1}= & \omega \\
f_{2}= & \frac{-d_{m} \omega+P_{m}+E_{m} Y_{m} V \sin \left(\delta-\delta_{m}-\theta_{m}\right)+E_{m}^{2} Y_{m} \sin \theta_{m}}{M}, \\
f_{3}= & \frac{-K_{q v 2} V^{2}-K_{q v} V+E_{0}^{\prime} Y_{0}^{\prime} V \cos \left(\delta+\theta_{0}^{\prime}\right)+E_{m} Y_{m} V \cos \left(\delta-\delta_{m}+\theta_{m}\right)-\left(Y_{0}^{\prime} \cos \theta_{0}^{\prime}+Y_{m} \cos \theta_{m}\right) V^{2}-Q_{0}-Q_{1}}{K_{q w}}, \\
f_{4}= & \frac{K_{q w}\left(-E_{0}^{\prime} Y_{0}^{\prime} V \sin \left(\delta+\theta_{0}^{\prime}\right)-E_{m} Y_{m} V \sin \left(\delta-\delta_{m}+\theta_{m}\right)+\left(Y_{0}^{\prime} \sin \theta_{0}^{\prime}+Y_{m} \sin \theta_{m}\right) V^{2}-P_{0}-P_{1}\right)}{T K_{q w} K_{p v}} \\
& -\frac{K_{p w}\left(E_{0}^{\prime} Y_{0}^{\prime} V \cos \left(\delta+\theta_{0}^{\prime}\right)+E_{m} Y_{m} V \cos \left(\delta-\delta_{m}+\theta_{m}\right)-\left(Y_{0}^{\prime} \cos \theta_{0}^{\prime}+Y_{m} \cos \theta_{m}\right) V^{2}-Q_{0}-Q_{1}\right)}{T K_{q w} K_{p v}} \\
& +\frac{K_{p w} K_{q v 2} V^{2}+\left(K_{p w} K_{q v}-K_{q w} K_{p v}\right) V}{T K_{q w} K_{p v}} .
\end{aligned}
$$

The desired value for system output is $V_{d}=1$ and the time scale separation sliding mode control input can be designed as

$$
\begin{aligned}
u & =\frac{1}{K_{p w}}\left[-K_{q w}\left(-E_{0}^{\prime} Y_{0}^{\prime} V \sin \left(\delta+\theta_{0}^{\prime}\right)\right.\right. \\
& -E_{m} Y_{m} V \sin \left(\delta-\delta_{m}+\theta_{m}\right) \\
& \left.+\left(Y_{0}^{\prime} \sin \theta_{0}^{\prime}+Y_{m} \sin \theta_{m}\right) V^{2}-P_{0}-P_{1}\right) \\
& +K_{p w}\left(E_{0}^{\prime} Y_{0}^{\prime} V \cos \left(\delta+\theta_{0}^{\prime}\right)\right. \\
& +E_{m} Y_{m} V \cos \left(\delta-\delta_{m}+\theta_{m}\right) \\
& \left.-\left(Y_{0}^{\prime} \cos \theta_{0}^{\prime}+Y_{m} \cos \theta_{m}\right) V^{2}-Q_{0}-Q_{1}\right) \\
& -K_{p w} K_{q v 2} V^{2}-\left(K_{p w} K_{q v}-K_{q w} K_{p v}\right) V \\
& \left.-T K_{q w} K_{p v} k\left(V-V_{d}\right)\right]+w .
\end{aligned}
$$

Two illustrative examples are provided to show the effectiveness, the robustness, and the superiority of proposed control method. The parameters for proposed controller are selected as $\varepsilon_{1}=0.01, \varepsilon_{2}=0.1$, and $k=10$.

In the first example, reactive power of constant PQ load is set as $Q_{1}=11.377$ to demonstrate the effectiveness of proposed controller in chaos suppression and nominal system performance recovery for studied power system. Since chaos is a precursor for voltage collapse in power system, an immediate control action is taken to suppress chaos in our simulation. The time responses of state variables under proposed controller and responding curves of nominal system are displayed in Figure 5. It is clear to see from Figure 5 that the trajectories of controlled system track the trajectories of nominal system and finally settle down to equilibrium state. The time response of proposed control input also converges to the nominal one, as illustrated in Figure 6.

The second example is demonstrated to show the robustness and superiority of the proposed control strategy. In this example, reactive power of constant PQ load slowly varies from 11.377 p.u. to 8.06 p.u. while other parameters keep no changes. Figures 7-13 compare the simulation results under proposed control scheme (time scale separation SMC) and two main disturbance estimation based sliding mode control 


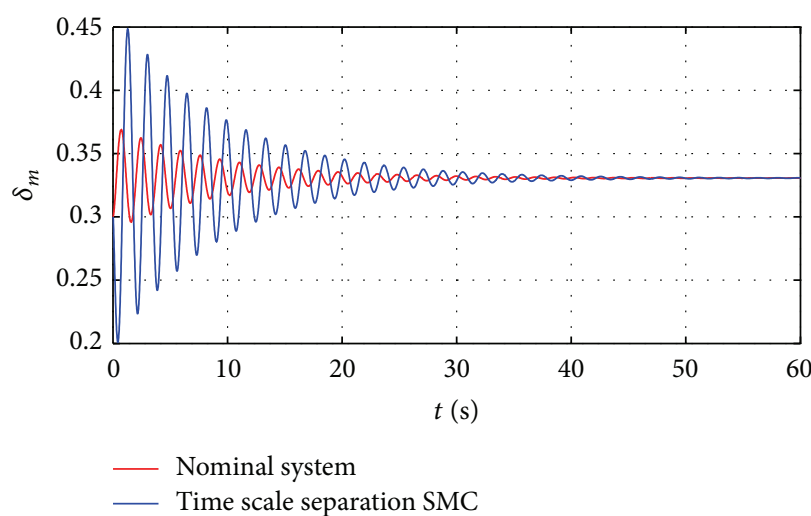

(a)

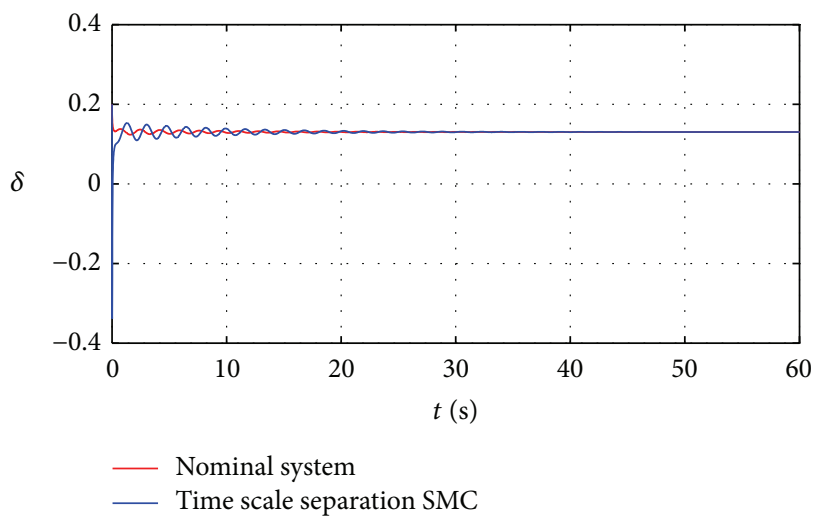

(c)

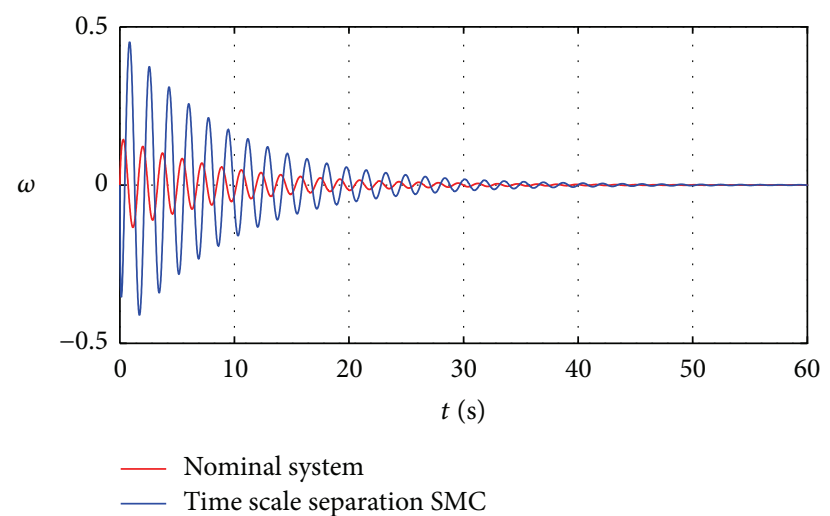

(b)

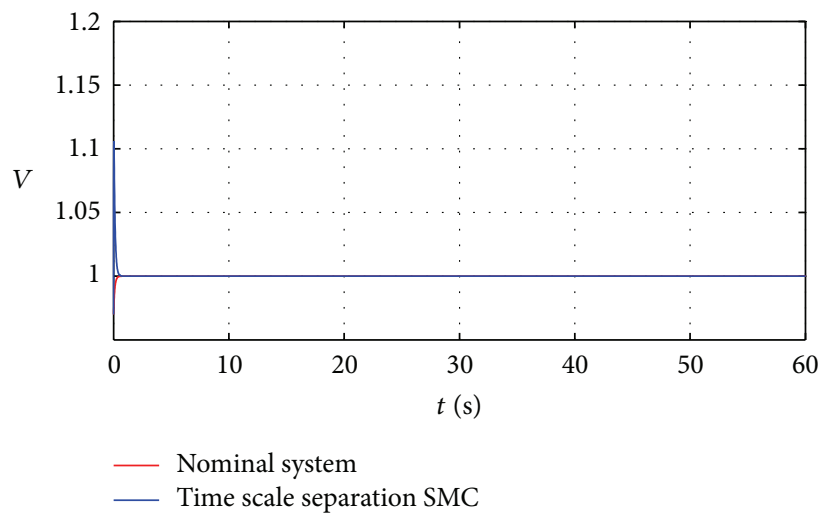

(d)

Figure 5: Time responses of nominal system and chaotic power system under proposed control scheme (a) generator angle $\delta_{m}$, (b) frequency deviation $\omega$, (c) load angle $\delta$, and (d) load voltage $V$.

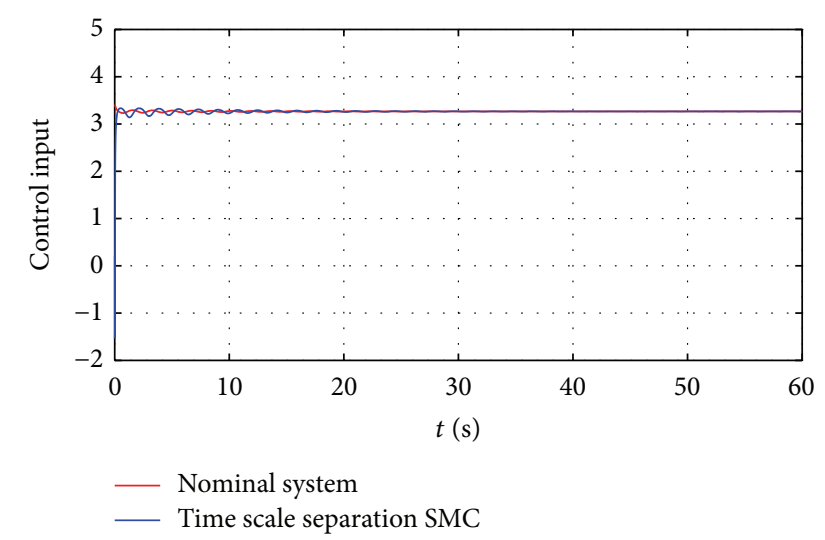

FIGURE 6: Time responses of nominal control input and proposed control input.

methods, that is, adaptive sliding mode control (adaptive SMC) and nonlinear disturbance observer based sliding mode control (NDOB based SMC). The control schemes proposed in [43] and [38] are borrowed as typical examples of adaptive SMC and NDOB based SMC, respectively, to make a comparison.

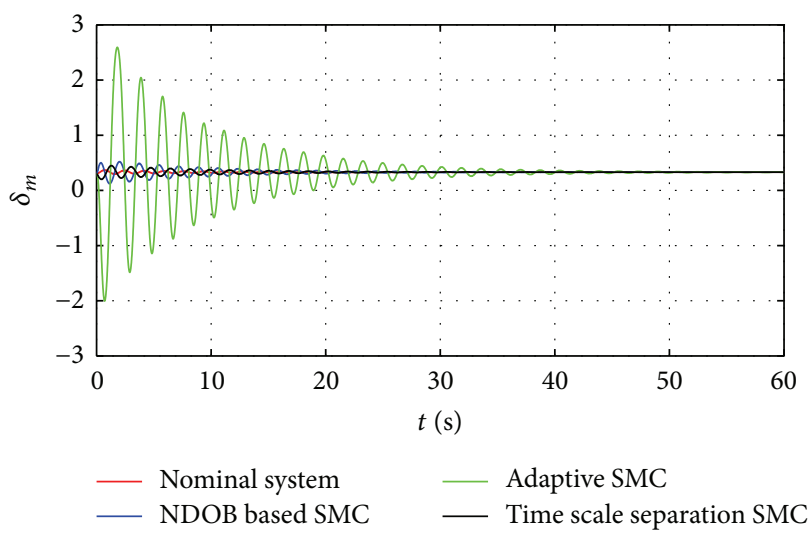

FIGURE 7: Time responses of generator angle in nominal system and disturbed power system under NDOB based SMC, adaptive SMC, and proposed time scale separation SMC.

Figures 7-10 display the time responses of state variables in disturbed power system under these three control methods, where red lines, blue lines, green lines, and black lines depict the responses of state variables in nominal system, system under NDOB based SMC, system under adaptive SMC, and system under the proposed control 


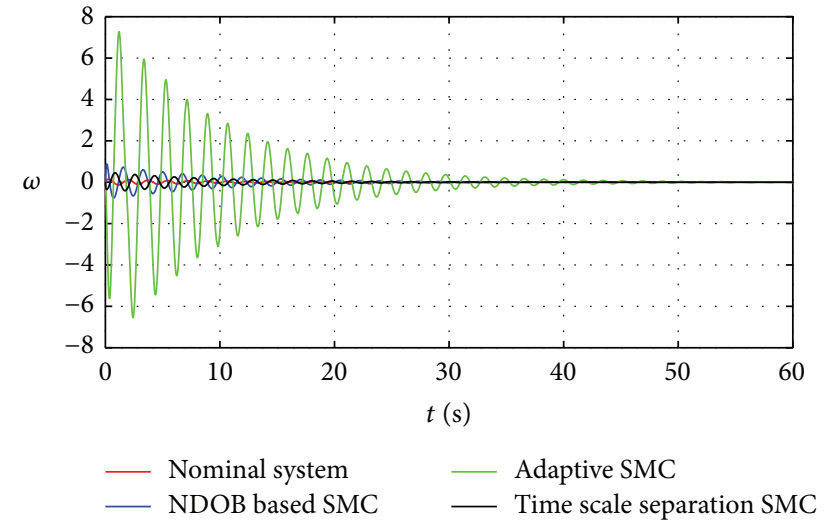

FIGURE 8: Time responses of frequency deviation in nominal system and disturbed power system under NDOB based SMC, adaptive SMC, and proposed time scale separation SMC.

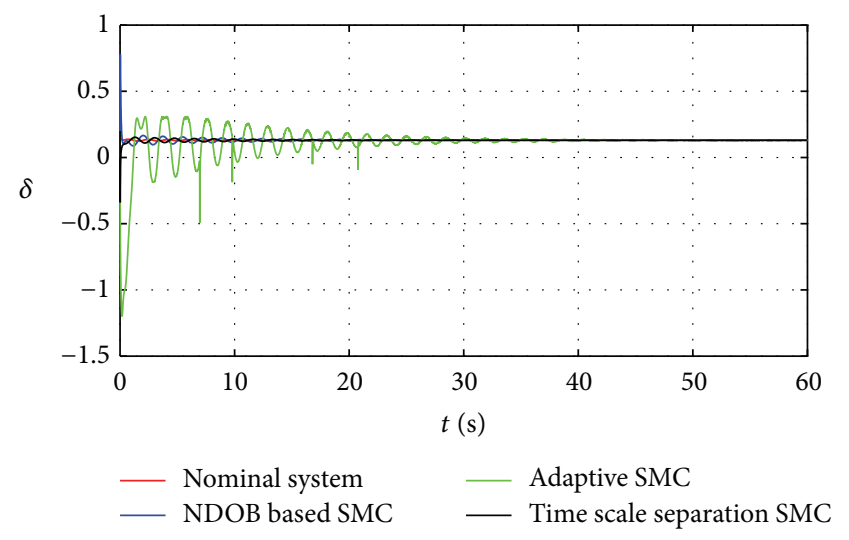

Figure 9: Time responses of load angle in nominal system and disturbed power system under NDOB based SMC, adaptive SMC, and proposed time scale separation SMC.



FIGURE 10: Time responses of load voltage in nominal system and disturbed power system under NDOB based SMC, adaptive SMC, and proposed time scale separation SMC.

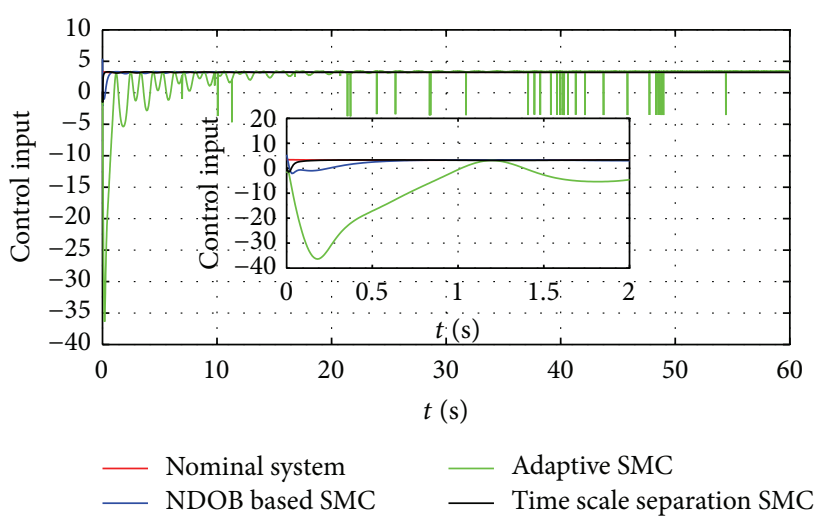

FIGURE 11: Time responses of nominal control input and NDOB based SMC, adaptive SMC, and proposed time scale separation SMC.

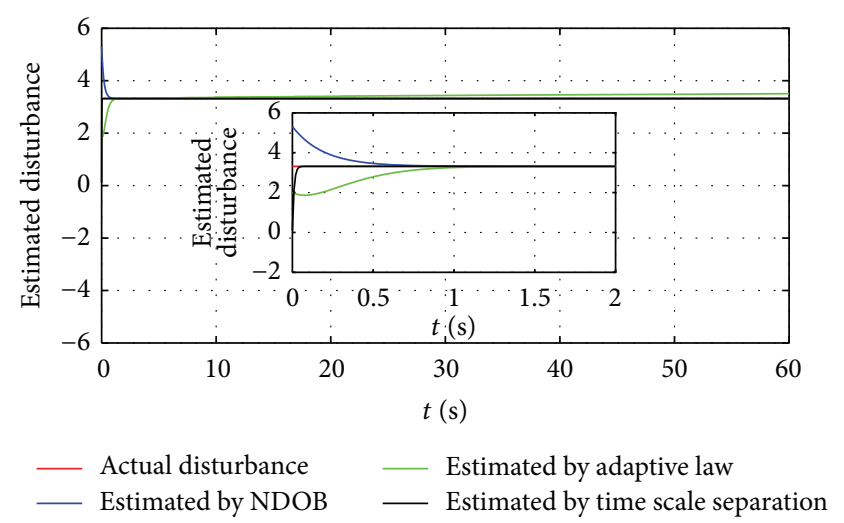

FIGURE 12: Time responses of actual disturbance and estimated disturbance by NDOB, adaptive law, and time scale separation.

scheme, respectively. It can be seen from these figures that the three control schemes can not only stabilize the state variables to their stable states, but also achieve performance recovery of nominal system. In addition, the amplitude of system transient response under proposed control scheme is smaller than that under the other two control schemes. The control actions for three control schemes are given in Figure 11, where red line, blue line, green line, and black line, respectively, correspond to nominal control input, NDOB based SMC input, adaptive SMC input, and the proposed control input. The inset of Figure 11 exhibits these control inputs extracted from $t=0 \mathrm{~s}$ to $t=2 \mathrm{~s}$. It is apparent from Figure 11 that adaptive SMC action produces serious chattering behavior due to overadaptation switching gain, whereas the proposed control input and NDOB based SMC input are smooth and no chattering is observed in these two control schemes. The corresponding sliding surface is also plotted in Figure 13, where red line, blue line, green line, and black line sketch the sliding surface of nominal control, NDOB based SMC, adaptive SMC, and the proposed control scheme, respectively. It can be seen from Figure 13 that the system states under proposed control scheme reach the sliding surface faster than the other two control schemes 


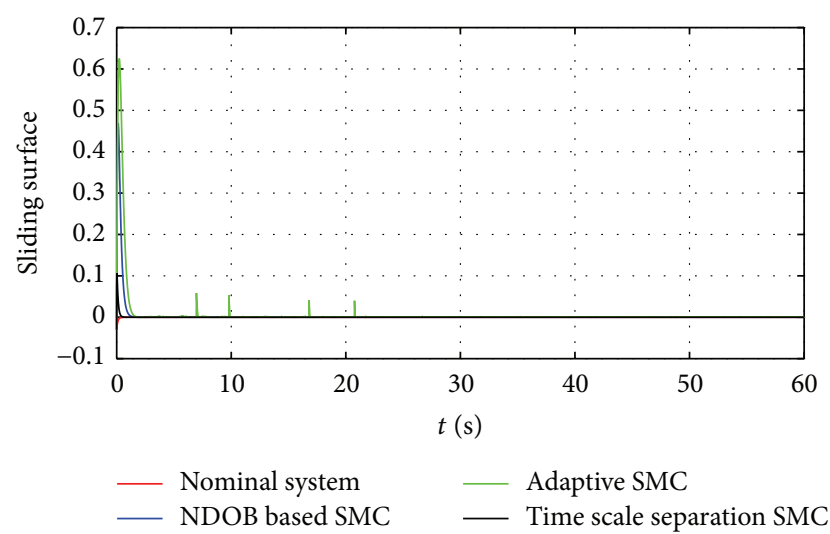

FIGURE 13: Time responses of sliding surface in nominal system and disturbed power system under NDOB based SMC, adaptive SMC, and proposed time scale separation SMC.

and keep on the sliding surface for the subsequent time, while the system states under adaptive SMC cannot stay on the sliding surface and shows severe chattering. The estimated disturbance and actual disturbance are depicted in Figure 12, where red line, blue line, green line, and black line show the actual disturbance and estimated disturbance by NDOB, adaptive law, and time scale separation. The inset of Figure 12 demonstrates the disturbances extracted from $t=0 \mathrm{~s}$ to $t=2 \mathrm{~s}$. It can be clearly observed that the proposed control scheme achieves much faster disturbance estimation than the other two control schemes. Meanwhile, it can be found that the disturbance estimated by adaptive SMC is slightly higher than the actual disturbance value, which follows the overadaptation problem exists in adaptive SMC. From these figures, we can see that the proposed control scheme achieves faster disturbance estimation and has faster convergence rate and smaller oscillatory response in comparison with the other two control schemes. Based on above discussion, it can be concluded that the proposed control method is robust against disturbance and outperforms adaptive SMC and NDOB based SMC in chattering alleviation effect and system transient response.

\section{Conclusions}

In this paper, a time scale separation sliding mode control scheme is presented for a class of disturbed nonlinear systems to eliminate chattering in sliding mode control. The proposed control scheme employs time scale separation design to achieve fast and exact disturbance estimation as well as nominal system and nominal control input performance recovery. The discontinuous switching term is replaced by continuous function, thereby eliminating chattering completely. Compared with existing results on disturbance estimation based sliding mode control methods, the proposed control strategy achieves better chattering alleviation effect and provides better transient response. The proposed control method is applied to suppress chaos in power system and simulation results verify its effectiveness, robustness, and superiority in power system chaos suppression.

\section{Appendix}

This appendix provides full detail of the derivation process for (35) and (36).

For (35): using (20), (24)-(26), (31), and (33), we have

$$
\begin{aligned}
\left\|\frac{\mathrm{d}(\alpha(x) \widetilde{w})}{\mathrm{d} t}\right\| \leq\left\|\frac{\mathrm{d}(\alpha(x))}{\mathrm{d} t}\right\|\|\widetilde{w}\|+\left\|\alpha(x) \frac{\mathrm{d} \widetilde{w}}{\mathrm{~d} t}\right\| \\
=\left\|\frac{\mathrm{d}(\alpha(x))}{\mathrm{d} t}\right\|\|\widetilde{w}\|+\frac{1}{\varepsilon_{1}}\left\|\alpha(x)\left(\alpha(x)^{-1} l+u\right)\right\| \\
\leq \quad L_{3}\|\widetilde{w}\| \\
\quad+\frac{1}{\varepsilon_{1}}\left\|\alpha(x)\left(\alpha(x)^{-1} l+u+w+d-w-d\right)\right\| \\
=L_{3}\|\widetilde{w}\| \\
\quad+\frac{1}{\varepsilon_{1}}\|l+\alpha(x)(u+w+d)-\alpha(x)(w+d)\| \\
\leq L_{3}\|\widetilde{w}\|+\frac{1}{\varepsilon_{1}}\|\widetilde{l}\|+\frac{L_{1}}{\varepsilon_{1}}\|\widetilde{w}\| \leq L_{5}\|\widetilde{w}\|+L_{6}\|\widetilde{l}\|,
\end{aligned}
$$

where $L_{5}=L_{3}+L_{1} / \varepsilon_{1}$ and $L_{6}=1 / \varepsilon_{1}$.

For (36): define

$$
\tilde{f}(x)=L_{f} h(x)+\alpha(x) u .
$$

Substituting (14) into (A.2), one has

$$
\|\tilde{f}(x)\|=k\|s\| .
$$

From (A.3) and (31), we have

$$
\begin{aligned}
\|\dot{s}\| & =\left\|L_{f} h(x)+\alpha(x) u+\alpha(x)(w+d)\right\| \\
& \leq\left\|L_{f} h(x)+\alpha(x) u\right\|+\|\alpha(x)(w+d)\| \\
& =\|\widetilde{f}(x)\|+\|\alpha(x) \widetilde{w}\| \leq\|\tilde{f}(x)\|+\|\alpha(x)\|\|\widetilde{w}\| \\
& \leq k\|s\|+L_{1}\|\widetilde{w}\| .
\end{aligned}
$$

Using (A.4) and (32), one obtains

$$
\begin{aligned}
\left\|\frac{\mathrm{d}(\alpha(x) u)}{\mathrm{d} t}\right\| & =\left\|\frac{\mathrm{d}(\alpha(x) u)}{\mathrm{d} s} \frac{\mathrm{d} s}{\mathrm{~d} t}\right\| \\
& \leq\left\|\frac{\mathrm{d}(\alpha(x) u)}{\mathrm{d} s}\right\|\left\|\frac{\mathrm{d} s}{\mathrm{~d} t}\right\| \\
& \leq L_{2}\left(k\|s\|+L_{1}\|\widetilde{w}\|\right) \\
& =L_{7}\|s\|+L_{8}\|\widetilde{w}\|,
\end{aligned}
$$

where $L_{7}=k L_{2}$ and $L_{8}=L_{1} L_{2}$.

\section{Conflict of Interests}

The authors declare that there is no conflict of interests regarding the publication of this paper. 


\section{Acknowledgments}

This project was supported by the National Natural Science Foundation of China under Grants nos. 51177117 and 51307130 and the Creative Research Groups Fund of the National Natural Science Foundation of China under Grant no. 51221005.

\section{References}

[1] W. Ji and V. Venkatasubramanian, "Hard-limit induced chaos in a fundamental power system model," International Journal of Electrical Power and Energy Systems, vol. 18, no. 5, pp. 279-295, 1996.

[2] M. L. Ma and F. H. Min, "Bifurcation behavior and coexisting motions in a time-delayed power system," Chinese Physics B, vol. 24, no. 3, Article ID 030501, 2015.

[3] D. Q. Wei and X. S. Luo, "Noise-induced chaos in singlemachine infinite-bus power systems," EPL, vol. 86, no. 5, Article ID 50008, 2009.

[4] D. Q. Wei, B. Zhang, D. Y. Qiu, and X. S. Luo, "Effect of noise on erosion of safe basin in power system," Nonlinear Dynamics, vol. 61, no. 3, pp. 477-482, 2010.

[5] Y.-H. Qin, X.-S. Luo, and D.-Q. Wei, "Random-phase-induced chaos in power systems," Chinese Physics B, vol. 19, no. 5, Article ID 0505111, 2010.

[6] H.-D. Chiang, C.-W. Liu, P. P. Varaiya, F. F. Wu, and M. G. Lauby, "Chaos in a simple power system," IEEE Transactions on Power Systems, vol. 8, no. 4, pp. 1407-1417, 1993.

[7] Z. J. Jing, D. S. Xu, Y. Chang, and L. N. Chen, "Bifurcations, chaos, and system collapse in a three node power system," International Journal of Electrical Power and Energy System, vol. 25, no. 6, pp. 443-461, 2003.

[8] Y. X. Yu, H. J. Jia, P. Li, and J. F. Su, "Power system instability and chaos," Electric Power Systems Research, vol. 65, no. 3, pp. 187-195, 2003.

[9] X.-Y. Wang and J.-M. Song, "Synchronization of the fractional order hyperchaos Lorenz systems with activation feedback control," Communications in Nonlinear Science and Numerical Simulation, vol. 14, no. 8, pp. 3351-3357, 2009.

[10] C. G. Li and G. R. Chen, "Chaos in the fractional order chen system and its control," Chaos, Solitons \& Fractals, vol. 22, no. 3, pp. 549-554, 2004.

[11] D. Q. Wei and X. S. Luo, "Passivity-based adaptive control of chaotic oscillations in power system," Chaos, Solitons \& Fractals, vol. 31, no. 3, pp. 665-671, 2007.

[12] X. Y. Wang, X. P. Zhang, and C. Ma, "Modified projective synchronization of fractional-order chaotic systems via active sliding mode control," Nonlinear Dynamics, vol. 69, no. 1-2, pp. 511-517, 2012.

[13] M. P. Aghababa, "Finite-time chaos control and synchronization of fractional-order nonautonomous chaotic (hyperchaotic) systems using fractional nonsingular terminal sliding mode technique," Nonlinear Dynamics, vol. 69, no. 1-2, pp. 247-261, 2012.

[14] H. Wang, Z.-Z. Han, Q.-Y. Xie, and W. Zhang, "Finite-time chaos synchronization of unified chaotic system with uncertain parameters," Communications in Nonlinear Science and Numerical Simulation, vol. 14, no. 5, pp. 2239-2247, 2009.

[15] S. T. Liu and P. Liu, "Adaptive anti-synchronization of chaotic complex nonlinear systems with unknown parameters," Nonlinear Analysis: Real World Applications, vol. 12, no. 6, pp. 30463055, 2011.
[16] D. Lin and X. Y. Wang, “Observer-based decentralized fuzzy neural sliding mode control for interconnected unknown chaotic systems via network structure adaptation," Fuzzy Sets and Systems, vol. 161, no. 15, pp. 2066-2080, 2010.

[17] Z.-G. Wu, P. Shi, H. Y. Su, and J. Chu, "Sampled-data fuzzy control of chaotic systems based on a T-S fuzzy model," IEEE Transactions on Fuzzy Systems, vol. 22, no. 1, pp. 153-163, 2014.

[18] Q. Y. Sun, Y. G. Wang, J. Yang, Y. Qiu, and H. G. Zhang, "Chaotic dynamics in smart grid and suppression scheme via generalized fuzzy hyperbolic model," Mathematical Problems in Engineering, vol. 2014, Article ID 761271, 7 pages, 2014.

[19] D. Lin, X. Y. Wang, F. Z. Nian, and Y. L. Zhang, "Dynamic fuzzy neural networks modeling and adaptive backstepping tracking control of uncertain chaotic systems," Neurocomputing, vol. 73, no. $16-18$, pp. $2873-2881,2010$.

[20] K. Hirasawa, X. F. Wang, J. Murata, J. L. Hu, and C. Z. Jin, "Universal learning network and its application to chaos control," Neural Networks, vol. 13, no. 2, pp. 239-253, 2000.

[21] P. Mercorelli, "An antisaturating adaptive preaction and a slide surface to achieve soft landing control for electromagnetic actuators," IEEE/ASME Transactions on Mechatronics, vol. 17, no. 1, pp. 76-85, 2012.

[22] B. Haus, P. Mercorelli, and N. Werner, "A robust adaptive selftuning sliding mode control for a hybrid actuator in camless internal combustion engines," in Advances and Applications in Sliding Mode Control Systems, vol. 576 of Studies in Computational Intelligence, pp. 107-136, Springer, 2015.

[23] M. L. Corradini, G. Ippoliti, S. Longhi, and G. Orlando, "A quasi-sliding mode approach for robust control and speed estimation of PM synchronous motors," IEEE Transactions on Industrial Electronics, vol. 59, no. 2, pp. 1096-1104, 2012.

[24] S.-C. Tan, Y. M. Lai, and C. K. Tse, "General design issues of sliding-mode controllers in DC-DC converters," IEEE Transactions on Industrial Electronics, vol. 55, no. 3, pp. 1160-1174, 2008.

[25] E. Nechadi, M. N. Harmas, A. Hamzaoui, and N. Essounbouli, "A new robust adaptive fuzzy sliding mode power system stabilizer," International Journal of Electrical Power and Energy Systems, vol. 42, no. 1, pp. 1-7, 2012.

[26] J. A. Burton and A. S. I. Zinober, "Continuous approximation of variable structure control," International Journal of Systems Science, vol. 17, no. 6, pp. 875-885, 1986.

[27] M.-S. Chen, Y.-R. Hwang, and M. Tomizuka, "A statedependent boundary layer design for sliding mode control," IEEE Transactions on Automatic Control, vol. 47, no. 10, pp. 1677-1681, 2002.

[28] Y. J. Wu, Y. M. Liu, and W. L. Zhang, "A discrete-time chattering free sliding mode control with multirate sampling method for flight simulator," Mathematical Problems in Engineering, vol. 2013, Article ID 865493, 8 pages, 2013.

[29] Z. W. Qiao, T. N. Shi, Y. D. Wang, Y. Yan, C. L. Xia, and X. N. He, "New sliding-mode observer for position sensorless control of permanent-magnet synchronous motor," IEEE Transactions on Industrial Electronics, vol. 60, no. 2, pp. 710-719, 2013.

[30] M. P. Aghababa and M. E. Akbari, "A chattering-free robust adaptive sliding mode controller for synchronization of two different chaotic systems with unknown uncertainties and external disturbances," Applied Mathematics and Computation, vol. 218, no. 9, pp. 5757-5768, 2012.

[31] F.-J. Lin and S.-L. Chiu, "Adaptive fuzzy sliding-mode control for PM synchronous servo motor drives," IEE ProceedingsControl Theory and Applications, vol. 145, no. 1, pp. 63-72, 1998. 
[32] S. Mobayen, "An adaptive chattering-free PID sliding mode control based on dynamic sliding manifolds for a class of uncertain nonlinear systems," Nonlinear Dynamics, vol. 82, no. 1-2, pp. 53-60, 2015.

[33] H. Lee and V. I. Utkin, "Chattering suppression methods in sliding mode control systems," Annual Reviews in Control, vol. 31, no. 2, pp. 179-188, 2007.

[34] B. L. Cong, Z. Chen, and X. D. Liu, "Disturbance observerbased adaptive integral sliding mode control for rigid spacecraft attitude maneuvers," Proceedings of the Institute of Mechanical Engineers G-Journal of Aerospace Engineering, vol. 227, no. 10, pp. 1660-1671, 2013.

[35] F. Plestan, Y. B. Shtessel, V. Bregeault, and A. Poznyak, "New methodologies for adaptive sliding mode control," International Journal of Control, vol. 83, no. 9, pp. 1907-1919, 2010.

[36] X. G. Zhang, L. Z. Sun, K. Zhao, and L. Sun, "Nonlinear speed control for PMSM system using sliding-mode control and disturbance compensation techniques," IEEE Transactions on Power Electronics, vol. 28, no. 3, pp. 1358-1365, 2013.

[37] J. Yang, S. H. Li, J. Y. Su, and X. H. Yu, “Continuous nonsingular terminal sliding mode control for systems with mismatched disturbances," Automatica, vol. 49, no. 7, pp. 2287-2291, 2013.

[38] M. Chen and W.-H. Chen, "Sliding mode control for a class of uncertain nonlinear system based on disturbance observer," International Journal of Adaptive Control and Signal Processing, vol. 24, no. 1, pp. 51-64, 2010.

[39] X. L. Cheng, G. J. Tang, P. Wang, and L. H. Liu, "Predictive sliding mode control for attitude tracking of hypersonic vehicles using fuzzy disturbance observer," Mathematical Problems in Engineering, vol. 2015, Article ID 727162, 13 pages, 2015.

[40] P. Mercorelli, "A two-stage sliding-mode high-gain observer to reduce uncertainties and disturbances effects for sensorless control in automotive applications," IEEE Transactions on Industrial Electronics, vol. 62, no. 9, pp. 5929-5940, 2015.

[41] D. Won, W. Kim, D. Shin, and C. C. Chung, "High-gain disturbance observer-based backstepping control with output tracking error constraint for electro-hydraulic systems," IEEE Transactions on Control Systems Technology, vol. 23, no. 2, pp. 787-795, 2015.

[42] A. Chakrabortty and M. Arcak, "Time-scale separation redesigns for stabilization and performance recovery of uncertain nonlinear systems," Automatica, vol. 45, no. 1, pp. 34-44, 2009.

[43] Q. L. Hu, "Sliding mode maneuvering control and active vibration damping of three-axis stabilized flexible spacecraft with actuator dynamics," Nonlinear Dynamics, vol. 52, no. 3, pp. 227-248, 2008.

[44] D. Q. Zhang and S. K. Panda, "Chattering-free and fast-response sliding mode controller," IEE Proceedings-Control Theory and Applications, vol. 146, no. 2, pp. 171-177, 1999.

[45] Y. Xu, "Chattering free robust control for nonlinear systems," IEEE Transactions on Control Systems Technology, vol. 16, no. 6, pp. 1352-1359, 2008.

[46] P. V. Kokotović and H. K. Khalil, Singular Perturbations in Systems and Control, IEEE Press, New York, NY, USA, 1986.

[47] I. Dobson and H.-D. Chiang, "Towards a theory of voltage collapse in electric power systems," Systems \& Control Letters, vol. 13, no. 3, pp. 253-262, 1989.

[48] K. Walve, "Modelling of power system components at severe disturbances," CIGRE Report, 1986.
[49] A. Wolf, J. B. Swift, H. L. Swinney, and J. A. Vastano, "Determining Lyapunov exponents from a time series," Physica D, vol. 16, no. 3, pp. 285-317, 1985.

[50] M. S. Saad, M. A. Hassouneh, E. H. Abed, and A.-A. Edris, "Delaying instability and voltage collapse in power systems using SVCs with washout filter-aided feedback," in Proceedings of the American Control Conference, pp. 4357-4362, IEEE, Portland, Ore, USA, June 2005.

[51] I. M. Ginarsa, A. Soeprijanto, and M. H. Purnomo, "Controlling chaos and voltage collapse using an ANFIS-based composite controller-static var compensator in power systems," International Journal of Electrical Power and Energy Systems, vol. 46, no. 1, pp. 79-88, 2013.

[52] D. C. Liaw, S. T. Chang, and Y. H. Huang, "Voltage tracking design for electric power systems via SMC approach," in Proceedings of the 48th IEEE Conference on Decision and Control, 28th Chinese Control Conference (CDC/CCC '09), pp. 78547859, Shanghai, China, December 2009. 


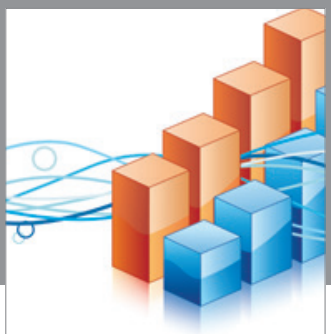

Advances in

Operations Research

vatem alat4

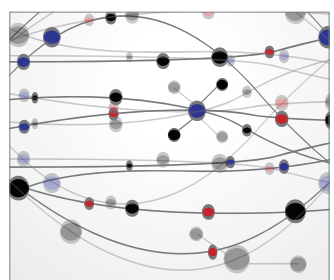

\section{The Scientific} World Journal



Algebra

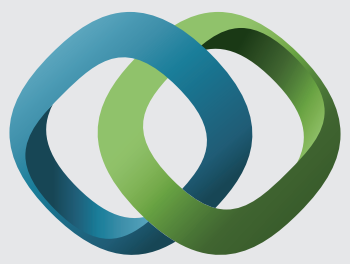

\section{Hindawi}

Submit your manuscripts at

http://www.hindawi.com
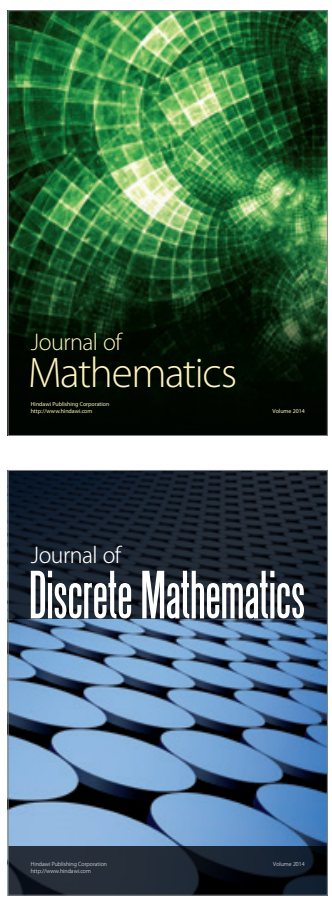



Mathematical Problems in Engineering
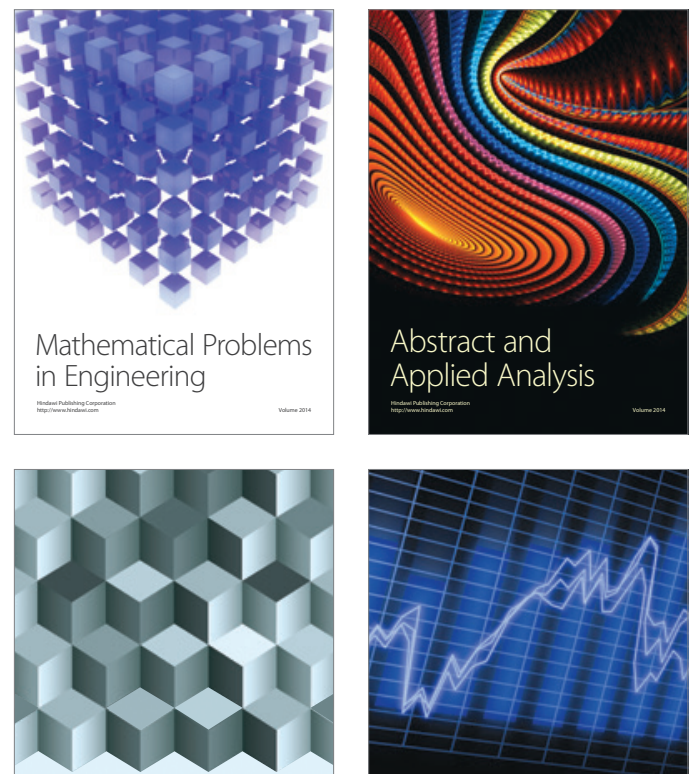

Journal of

Function Spaces

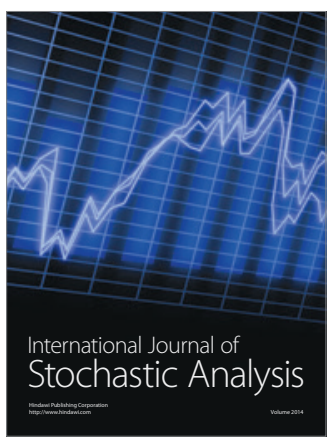

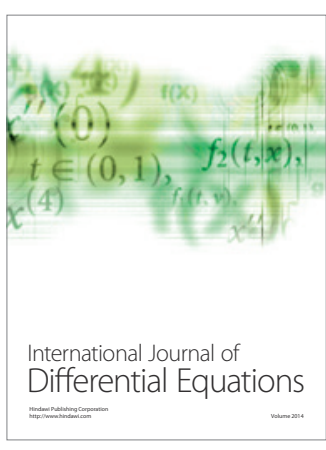
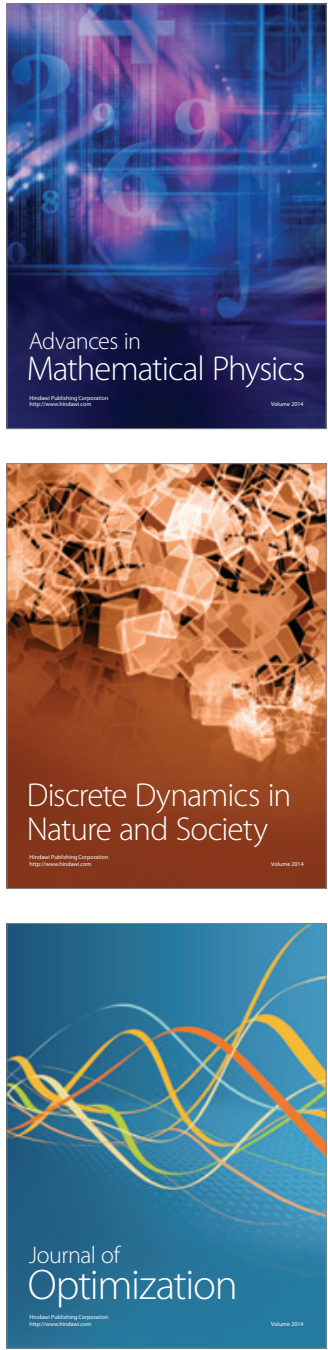